\title{
On semi-invariant submanifolds of a generalized Kenmotsu manifold admitting a semi-symmetric non-metric connection
}

\author{
Aysel Turgut Vanli, Ramazan Sari \\ Department of Mathematics, Faculty of Arts and Sciences, Gazi University, 06500 Ankara, Turkey \\ Email address: \\ avanli@gazi.edu.tr (A. T. Vanli), ramazansr@gmail.com (R. Sari)
}

\section{To cite this article:}

Aysel Turgut Vanli, Ramazan Sari. On Semi-Invariant Submanifolds of a Generalized Kenmotsu Manifold Admitting a Semi-Symmetric Non-Metric Connection. Pure and Applied Mathematics Journal. Special Issue: Applications of Geometry. Vol. 4, No. 1-2, 2015 , pp. 14-18. doi: 10.11648/j.pamj.s.2015040102.14

\begin{abstract}
In this paper, semi-invariant submanifolds of a generalized Kenmotsu manifold endowed with a semi-symmetric non-metric connection are studied. Necessary and sufficient conditions are given on a submanifold of a generalized Kenmotsu manifold to be semi-invarinat submanifold with semi-symmetric non-metric connection. Morever, we studied the integrability condition of the distribution on semi-invariant submanifolds of generalized Kenmotsu manifold with semi-symmetric non-metric connection.
\end{abstract}

Keywords: Generalized Kenmotsu Manifolds, Semi-Invariant Submanifolds, Semi-Symmetric Non-Metric Connection

\section{Introduction}

In 1963, Yano [11] introduced an f-structure on a $\mathrm{C}^{\infty}$ $\mathrm{m}$-dimensional manifold $\mathrm{M}$, defined by a non-vanishing tensor field $\varphi$ of type $(1,1)$ which satisfies $\varphi^{3}+\varphi=0$ and has constant rank r. It is know that in this case $r$ is even, $\mathrm{r}=2 \mathrm{n}$. Moreover, TM splits into two complementary subbundles $\operatorname{Im} \varphi$ and $\operatorname{ker} \varphi$ and the restriction of $\varphi$ to $\operatorname{Im} \varphi$ determines a complex structure on such subbundle. It is also known that the existence of an f-structure on $M$ is equivalent to a reduction of the structure group to $U(n) \times O(s)$

[1] where $s=m-2 n$.

In [2] , K. Kenmotsu has introduced a Kenmotsu manifold. In [9], present autours have introduced a generalized Kenmotsu manifold.

Semi-invariant submanifolds are studied by some authours (for examples, M. Kobayashi [3], B. Prasad [6] and B.B. Sinha, A.K. Srivastava [7]). In [5] S. A. Nirmala and R.C. Mangala have introduced a semi-symmetric non-metric connection, they studied some properties of the curvature tensor with respect to the semi-symmetric non-metric connection.

Let $\nabla$ be a linear connection in a $n$-dimensional differentiable manifold $\mathrm{M}$. The torsion tensor $\mathrm{T}$ of $\nabla$ is given by

$$
T(X, Y)=\nabla_{X} Y-\nabla_{Y} X-[X, Y]
$$

The connection $\nabla$ is symmetric if torsion tensor $\mathrm{T}$ vanishes, othervise it is non-symmetric. A lineer connection $\nabla$ is said to be semi-symmetric connection if it torsion tensor $\mathrm{T}$ is of the form

$$
T(X, Y)=\eta(Y) X-\eta(X) Y
$$

where $\eta$ is a 1 -form. The connection $\nabla$ is metric connection if there is a Riemannian metric $g$ in M such that $\nabla g=0$, otherwise it is non-metric. It is well known that a linear connection is symmetric and metric if it is the Levi-Civita connection.

The paper is organized as follows : In section 2, we give a brief introduction of generalized Kenmotsu manifold. We defined a generalized Kenmotsu manifold with a semi-symmetric non-metric connection. In section 3, we give some basic results for semi-invariant submanifolds of generalized Kenmotsu manifold with a semi-symmetric non-metric connection. In last section, we obtained some necessary and sufficient conditions for integrability of certain distributions on semi-invariant submanifolds of generalized 
Kenmotsu manifold with a semi-symmetric non-metric connection.

\section{Preliminaries}

In [4], a $(2 \mathrm{n}+\mathrm{s})$-dimensional differentiable manifold $M$ is called metric $f$-manifold if there exist an $(1,1)$-type tensor field $\varphi$, s-vector fields $\xi_{1}, \ldots, \xi_{s}$, s 1 -forms $\eta^{1}, \ldots, \eta^{s}$ and a Riemannian metric $\mathrm{g}$ on $M$ such that

$$
\begin{gathered}
\varphi^{2}=-I+\sum_{i=1}^{s} \eta^{i} \otimes \xi_{i}, \quad \eta^{i}\left(\xi_{j}\right)=\delta_{i j} \\
g(\varphi X, \varphi Y)=g(X, Y)-\sum_{i=1}^{s} \eta^{i}(X) \eta^{i}(Y),
\end{gathered}
$$

for any $X, Y \in \Gamma(T M), i, j \in\{1, \ldots, s\}$. In addition, we have

$$
\eta^{i}(X)=g\left(X, \xi_{i}\right), g(X, \varphi Y)=-g(\varphi X, Y) .
$$

Then, a 2 -form $\Phi$ is defined by $\Phi(X, Y)=g(X, \varphi Y)$, for any $X, Y \in \Gamma(T M)$, called the fundamental 2 -form. Moreover, a framed metric manifold is normal if

$$
[\varphi, \varphi]+2 \sum_{i=1}^{s} d \eta^{i} \otimes \xi_{i}=0
$$

where $[\varphi, \varphi]$ is denoting the Nijenhuis tensor field associated to $\varphi$.

In [10], let $M(2 n+s)$-dimensional metric $f$-manifold. If there exists 2 -form $\Phi$ such that $\eta^{1} \wedge \ldots \wedge \eta^{s} \wedge \Phi^{n} \neq 0$ on $M$, then $M$ is called an almost $s$-contact metric structure.

The almost $s$-contact metric manifold $\bar{M}$ is called a generalized Kenmotsu manifold if it satisfies the condition

$$
\left(\overline{\bar{\nabla}}_{X} \varphi\right) Y=\sum_{i=1}^{s}\left\{g(\varphi X, Y) \xi_{i}-\eta^{i}(Y) \varphi X\right\}
$$

where $\overline{\bar{\nabla}}$ denotes the Riemannian connection with respect to g [9].

From the formula (4) we have

$$
\overline{\bar{\nabla}}_{X} \xi_{i}=-\varphi^{2} X
$$

Definition 2.1 An $(2 n+s)$-dimensional Riemannian submanifold $M$ of a generalized Kenmotsu manifold $\bar{M}$ is called a semi-invariant submanifold if $\xi_{i}$ are tangent to $\bar{M}$ and there exists on $M$ a pair of orthogonal distribution $\left\{D, D^{\perp}\right\}$ such that

(i) $T M=D \oplus D^{\perp} \oplus \operatorname{Sp}\left\{\xi_{1}, \ldots, \xi_{s}\right\}$.

(ii) The distribution $D$ is invariant under $\boldsymbol{\varphi}$, that is $\varphi D_{x}=D_{x}$, for all $x \in M$

(iii) The distribution $D^{\perp}$ is anti-invariant under $\varphi$, that is $\varphi D_{x}^{\perp} \subset T_{x} M^{\perp}$, for all $x \in M$, where $T_{x} M$ and
$T_{x} M^{\perp}$ are the tangent space of $\mathrm{M}$ at $x$.

The distribution $D$ (resp. $D^{\perp}$ ) is called horizontal (resp. vertical) distribution. A semi-invariant submanifold $M$ is said to be an invariant (resp. anti-invariant) submanifold if we have $D_{x}^{\perp}=\{0\} \quad$ (resp. $D_{x}=\{0\}$ ) for each $x \in M$. We say that $M$ is a proper semi-invariant submanifold, which is neither an invariant nor an anti-invariant submanifold.

Let $\overline{\bar{\nabla}}$ be the Levi-Civita connection of $\bar{M}$ with respect to the induced metric $\mathrm{g}$. Then Gauss and Weingarten formulas are given by

$$
\begin{aligned}
& \overline{\bar{\nabla}}_{X} Y=\nabla_{X}^{*} Y+h(X, Y) \\
& \overline{\bar{\nabla}}_{X} N=\nabla_{X}^{* \perp} N-A_{N} X
\end{aligned}
$$

for any $X, Y \in \Gamma(T M)$ and $N \in \Gamma\left(T M^{\perp}\right) \cdot \nabla^{\perp}$ is the connection in the normal bundle, $h$ is the second fundamental from of $\bar{M}$ and $A_{N}$ is the Weingarten endomorphism associated with $N$. The second fundamental form $h$ and the shape operator $A$ related by

$$
g(h(X, Y), N)=g\left(A_{N} X, Y\right) .
$$

Now, a semi-symmetric non-metric connection $\bar{\nabla}$ is defined as

$$
\bar{\nabla}_{X} Y=\overline{\bar{\nabla}}_{X} Y+\sum_{i=1}^{s} \eta^{i}(Y) X
$$

such that

$$
\left(\bar{\nabla}_{X} g\right)(Y, Z)=-\sum_{i=1}^{s}\left\{g\left((X, Y) \eta^{i}(Z)+g(X, Z) \eta^{i}(Y)\right\}\right.
$$

for any $X, Y \in T M$, where $\bar{\nabla}$ is induced connection on $M$.

From (4) and (9), we have

$$
\left(\bar{\nabla}_{X} \varphi\right) Y=\sum_{i=1}^{s}\left\{g(\varphi X, Y) \xi_{i}-2 \eta^{i}(Y) \varphi X\right\}
$$

which is condition for almost $s$-contact metric manifold to be generalized Kenmotsu manifold with semi-symmetric non-metric connection.

Corollary 2.2 Let $M$ be a semi-invariant submanifold of a generalized Kenmotsu manifold $\bar{M}$ with semi-symmetric non-metric connection, then

$$
\bar{\nabla}_{X} \xi_{j}=2 X-\sum_{j=1}^{s} \eta^{j}(X) \xi_{j}
$$

for all $X, Y \in T M$.

Proof. Putting $Y=\xi_{j}$ and $Z=\xi_{j}$ in (10)

$$
X\left[g\left(\xi_{j}, \xi_{j}\right)\right]-g\left(\bar{\nabla}_{X} \xi_{j}, \xi_{j}\right)-g\left(\xi_{j}, \bar{\nabla}_{X} \xi_{j}\right)=2 \eta^{j}(X) \text {. So }
$$




$$
g\left(\bar{\nabla}_{X} \xi_{j}, \xi_{j}\right)=\eta^{j}(X)
$$

Now, using (11)

$$
\left(\bar{\nabla}_{X} \varphi\right) \xi_{j}=\sum_{i=1}^{s}\left\{g\left(\varphi X, \xi_{j}\right) \xi_{i}-2 \eta^{i}\left(\xi_{j}\right) \varphi X\right\}
$$

or

$$
-\varphi \bar{\nabla}_{X} \xi_{j}=-2 \varphi X
$$

from (1) and (13)

$$
\bar{\nabla}_{X} \xi_{j}-\sum_{j=1}^{s} \eta^{j}\left(\bar{\nabla}_{X} \xi_{j}\right) \xi_{j}=-2\left(-X+\sum_{j=1}^{s} \eta^{j}(X) \xi_{j}\right) .
$$

We denote by same symbol $g$ both metrices on $\bar{M}$ and $M$. Let $\bar{\nabla}$ be the semi-symmetric non-metric connection on $\bar{M}$ and $\nabla$ be the induced connection on $M$ with respect to unit normal $N$. Then,

$$
\bar{\nabla}_{X} Y=\nabla_{X} Y+m(X, Y)
$$

where $m$ is a tensor field of type $(0,2)$ on semi-invariant submanifold $M$. Using (6) and (9) we have,

$$
\nabla_{X} Y+m(X, Y)=\nabla_{X}^{*} Y+h(X, Y)+\eta^{i}(Y) X .
$$

So equation tangential and normal components from both the sides, we get

$$
m(X, Y)=h(X, Y)
$$

and

$$
\nabla_{X} Y=\nabla_{X}^{*} Y+\sum_{i=1}^{s} \eta^{i}(Y) X
$$

From (15) and (7)

$$
\begin{aligned}
\nabla_{X} N & =\nabla_{X}^{*} N+\sum_{i=1}^{s} \eta^{i}(N) X \\
& =-A_{N} X+\sum_{i=1}^{s} \eta^{i}(N) X \\
& =\left(-A_{N}+a\right) X
\end{aligned}
$$

where $a=\sum_{i=1}^{s} \eta_{i}(N)$ is a function on $M$.

Now, Gauss and Weingarten formulas for a semi-invariant submanifolds of a generalized Kenmotsu manifold with a semi-symmetric non-metric connection is

$$
\bar{\nabla}_{X} Y=\nabla_{X} Y+h(X, Y)
$$

and

$$
\bar{\nabla}_{X} N=\left(-A_{N}+a\right) X+\nabla_{X}^{\perp} N
$$

for all $X, Y \in \Gamma(T M), \quad N \in \Gamma\left(T M^{\perp}\right), \quad h \quad$ second fundamental form of $M$ and $A_{N}$ is the Weingarten endomorphism associated with $N$. The second fundamental form $h$ and the shape operator $A$ related by

$$
g(h(X, Y), N)=g\left(A_{N} X, Y\right) .
$$

The projection morphisms of $T M$ to $D$ and $D^{\perp}$ are denoted by $P$ and $Q$ respectively. For any $X, Y \in \Gamma(T M)$ and $N \in \Gamma\left(T M^{\perp}\right)$, we have

$$
X=P X+Q X+\sum_{i=1}^{s} \eta^{i}(X) \xi_{i}
$$

and

$$
\varphi N=B N+C N
$$

where $B N$ (resp. $C N$ ) denotes the tangential (resp. normal) component of $\varphi N$.

Theorem 2.3 The connection induced on semi-invariant submanifolds of a generalized Kenmotsu manifold with semi-symmetric non-metric connection is also a semi-symmetric non-metric connection.

\section{Basic Results}

Lemma 3.1 Let M be a semi-invariant submanifold of a generalized Kenmotsu manifold $\bar{M}$ with semi-symmetric non-metric connection, then we have

$$
\begin{aligned}
\left(\bar{\nabla}_{X} \varphi\right) Y & =\left(\nabla_{X} P\right) Y+\left(-A_{Q Y}+a\right) X-B h(X, Y) \\
& +\left(\nabla_{X} Q\right) Y+h(X, P Y)-C h(X, Y)
\end{aligned}
$$

$$
\begin{aligned}
\left(\bar{\nabla}_{X} \varphi\right) N= & \left(\nabla_{X} B\right) N+\left(-A_{C N}+a\right) X+P\left(-A_{N}+a\right) X \\
& +\left(\nabla_{X} C\right) N+h(X, B N)+Q\left(-A_{N}+a\right) X
\end{aligned}
$$

for all $X, Y \in T M ; N \in \Gamma(T M)^{\perp}$ where $a=\sum_{i=1}^{s} \eta^{i}(C N)=0$.

Proof. Using (19) and (20), necessary arrangements are made to obtain the desired.

Lemma 3.2 Let $M$ be a semi-invariant submanifold of a generalized Kenmotsu manifold $\bar{M}$ with semi-symmetric non-metric connection, we have

$$
\begin{gathered}
\left(\nabla_{X} P\right) Y+\left(-A_{Q Y}+a\right) X-B h(X, Y)=-2 \sum_{i=1}^{s} \eta^{i}(Y) P X \\
\left(\nabla_{X} Q\right) Y+h(X, P Y)-C h(X, Y)=-2 \sum_{i=1}^{s} \eta^{i}(Y) Q X
\end{gathered}
$$




$$
\begin{gathered}
\left(\nabla_{X} B\right) N+\left(-A_{C N}+a\right) X+P\left(-A_{N}+a\right) X=0 \\
\left(\nabla_{X} C\right) N+h(X, B N)+Q\left(-A_{N}+a\right) X=0 \\
g(P X, Y)=0 \\
g(Q X, Y)=0
\end{gathered}
$$

for all $X, Y \in \Gamma(T M), \quad N \in \Gamma(T M)^{\perp}$.

Proof. Using (11) in (21) and (22), completes the proof.

Corollary 3.3 Let $M$ be a semi-invariant submanifold of a generalized Kenmotsu manifold $\bar{M}$ with semi-symmetric non-metric connection such that $\xi_{i} \in T M$, we have

$$
\begin{gathered}
\left(\nabla_{X} P\right) \xi_{i}=-2 P X \\
\left(\nabla_{X} Q\right) \xi_{i}=-2 Q X \\
\left(\nabla_{\xi_{i}} B\right) N=0, \nabla_{\xi_{i}} B=0 \\
\left(\nabla_{\xi_{i}} C\right) N=0, \nabla_{\xi_{i}} C=0 .
\end{gathered}
$$

For $X, Y \in \Gamma(T M)$, we put

$$
u(X, Y)=\nabla_{X} \varphi P Y-A_{\varphi Q Y} X .
$$

We begin with the following lemma.

Lemma 3.4 Let $M$ be a semi-invariant submanifold of a generalized Kenmotsu manifold $\bar{M}$ with semi-symmetric non-metric connection, then we have

$$
\begin{gathered}
P(u(X, Y))=\sum_{i=1}^{s}\left\{g(\varphi X, Y) P \xi_{i}-2 \eta^{i}(Y) \varphi P X\right\}+\varphi P \nabla_{X} Y \\
Q(u(X, Y))=\sum_{i=1}^{s}\left\{g(\varphi X, Y) Q \xi_{i}-2 \eta^{i}(Y) \varphi Q X\right\}+B h(X, Y) \\
\varphi Q \nabla_{X} Y+C h(X, Y)=h(X, \varphi P Y)+\nabla_{X}^{\perp} \varphi Q Y \\
\eta^{i}(u(X, Y))=g(\varphi X, Y)
\end{gathered}
$$

for all $X, Y \in \Gamma(T M)$.

Proof. Easily shown using (11), (16), (17), (19) and (20).

Lemma 3.5 Let $M$ be a semi-invariant submanifold of a generalized Kenmotsu manifold $\bar{M}$ with semi-symmetric non-metric connection such that $\xi_{i} \in T M$, we have

$$
\begin{gathered}
\nabla_{X} \xi_{i}=2 X-\sum_{i=1}^{s} \eta^{i}(X) \xi_{i}, h\left(X, \xi_{i}\right)=0 \\
\nabla_{\xi_{i}} \xi_{i}=0, \quad h\left(\xi_{i}, \xi_{i}\right)=0, \quad A_{N} \xi_{i}=0 .
\end{gathered}
$$

Proof. Using (12) and (15) for (22). And

$$
0=g\left(h\left(X, \xi_{i}\right), N\right)=g\left(A_{N} X, \xi_{i}\right)=g\left(A_{N} \xi_{i}, X\right) .
$$

\section{Integrability of Distribution on a Semi-Invariant Submanifolds a Generalized Kenmotsu Manifold with Semi-SymmetricNon-Metric Connection}

Theorem 4.1 Let $M$ be a semi-invariant submanifold of a generalized Kenmotsu manifold $\bar{M}$ with semi-symmetric non-metric connection. Then the distribution $D$ is integrable.

Proof. We have for all $X, Y \in \Gamma(D)$,

$$
\begin{aligned}
g\left([X, Y], \xi_{i}\right) & =g\left(\overline{\bar{\nabla}}_{X} Y, \xi_{i}\right)-g\left(\overline{\bar{\nabla}}_{Y} X, \xi_{i}\right) \\
& =-g\left(Y, \overline{\bar{\nabla}}_{X} \xi_{i}\right)+g\left(X, \overline{\bar{\nabla}}_{Y} \xi_{i}\right) .
\end{aligned}
$$

Using (9) and (12), we have

$$
\begin{aligned}
g\left([X, Y], \xi_{i}\right)=- & g\left(Y, \bar{\nabla}_{X} \xi_{i}-X\right)+g\left(X, \bar{\nabla}_{Y} \xi_{i}-Y\right) \\
=- & g\left(Y, 2 X-\sum_{i=1}^{s} \eta^{i}(X) \xi_{i}-X\right) \\
& +g\left(X, 2 Y-\sum_{i=1}^{s} \eta^{i}(Y) \xi_{i}-Y\right)
\end{aligned}
$$

$=0$.

So $\eta^{i}([X, Y])=0$ for $i=1,2, \ldots s$. Then, we have $[X, Y] \in D$.

Theorem 4.2 Let $M$ be a semi-invariant submanifold of a generalized Kenmotsu manifold $\bar{M}$ with semi-symmetric non-metric connection. The distribution $D \oplus S p\left\{\xi_{1}, \ldots, \xi_{s}\right\}$ is integrable if and only if

$$
h(X, \varphi Y)=h(\varphi X, Y)
$$

is satisfied.

Proof. Using (6) and (9), then

$$
\begin{aligned}
\varphi([X, Y])= & \varphi\left(\nabla_{X}^{*} Y-\nabla_{Y}^{*} X\right) \\
= & \varphi\left(\bar{\nabla}_{X} Y-h(X, Y)-\overline{\bar{\nabla}}_{Y} X+h(Y, X)\right) \\
= & \varphi\left(\bar{\nabla}_{X} Y-\sum_{i=1}^{s} \eta^{i}(Y) X-\bar{\nabla}_{Y} X+\sum_{i=1}^{s} \eta^{i}(X) Y\right) \\
= & \bar{\nabla}_{X} \varphi Y-\left(\bar{\nabla}_{X} \varphi\right) Y-\sum_{i=1}^{s} \eta^{i}(Y) \varphi X \\
& -\bar{\nabla}_{Y} \varphi X+\left(\bar{\nabla}_{Y} \varphi\right) X+\sum_{i=1}^{s} \eta^{i}(X) \varphi Y .
\end{aligned}
$$

For (11) and (16), we have 


$$
\begin{aligned}
\varphi([X, Y])= & \nabla_{X} \varphi Y-\nabla_{Y} \varphi X \\
& +\sum_{i=1}^{s}\left\{2 g(X, \varphi Y) \xi_{i}+\eta^{i}(Y) \varphi X-\eta^{i}(X) \varphi Y\right\} \\
& +h(X, \varphi Y)-h(\varphi X, Y)
\end{aligned}
$$

where $\varphi([X, Y])$ shows the component of $\nabla_{X} Y$ from the ortogonal complementary distribution of $D \oplus S p\left\{\xi_{1, \ldots, \ldots}, \xi_{s}\right\}$ in $M$. Then, we have $[X, Y] \in D \oplus S p\left\{\xi_{1}, \ldots, \xi_{s}\right\}$ if and only if $h(X, \varphi Y)=h(Y, \varphi X)$.

Theorem 4.3 Let $M$ be a semi-invariant submanifold of a generalized Kenmotsu manifold $\bar{M}$ with semi-symmetric non-metric connection. The distribution $D^{\perp} \oplus S p\left\{\xi_{1, \ldots,} \xi_{s}\right\}$ is integrable if and only if

$$
A_{\varphi X} Y=A_{\varphi Y} X
$$

is satisfied.

Proof. We have for all $X, Y \in \Gamma\left(D^{\perp}\right)$

$$
\begin{aligned}
g\left([X, Y], \xi_{i}\right) & =g\left(\overline{\bar{\nabla}}_{X} Y, \xi_{i}\right)-g\left(\overline{\bar{\nabla}}_{Y} X, \xi_{i}\right) \\
& =-g\left(Y, \overline{\bar{\nabla}}_{X} \xi_{i}\right)+g\left(X, \overline{\bar{\nabla}}_{Y} \xi_{i}\right) .
\end{aligned}
$$

Using (9) and (12), we have

$$
\begin{aligned}
g\left([X, Y], \xi_{i}\right)= & -g\left(Y, \bar{\nabla}_{X} \xi_{i}-X\right)+g\left(X, \bar{\nabla}_{Y} \xi_{i}-Y\right) \\
= & -g\left(Y, 2 X-\sum_{i=1}^{s} \eta^{i}(X) \xi_{i}-X\right) \\
& \quad+g\left(X, 2 Y-\sum_{i=1}^{s} \eta^{i}(Y) \xi_{i}-Y\right) \\
= & 0 .
\end{aligned}
$$

Using (6) and (9) then

$$
\begin{aligned}
\varphi([X, Y])= & \varphi\left(\nabla_{X}^{*} Y-\nabla_{Y}^{*} X\right) \\
= & \bar{\nabla}_{X} \varphi Y-\left(\bar{\nabla}_{X} \varphi\right) Y-\sum_{i=1}^{s} \eta^{i}(Y) \varphi X \\
& \quad-\bar{\nabla}_{Y} \varphi X+\left(\bar{\nabla}_{Y} \varphi\right) X+\sum_{i=1}^{s} \eta^{i}(X) \varphi Y .
\end{aligned}
$$

For (11) and (17), we have

$$
\begin{aligned}
\varphi([X, Y])= & \left(-A_{\varphi Y}+a\right) X+\nabla_{X}^{\perp} \varphi Y \\
& -\sum_{i=1}^{s}\left\{g(\varphi X, Y) \xi_{i}+2 \eta^{i}(Y) \varphi X\right\} \\
& -\left(-A_{\varphi X}+a\right) Y-\nabla_{Y}^{\perp} \varphi X \\
+ & \sum_{i=1}^{s}\left\{g(\varphi Y, X) \xi_{i}-2 \eta^{i}(X) \varphi Y\right. \\
+ & \left.\eta^{i}(X) \varphi Y-\eta^{i}(Y) \varphi X\right\}
\end{aligned}
$$

$$
\begin{gathered}
=\sum_{i=1}^{s}\left\{2 g(X, \varphi Y) \xi_{i}+\eta^{i}(Y) \varphi X-\eta^{i}(X) \varphi Y\right\} \\
+A_{\varphi X} Y-A_{\varphi Y} X+\nabla_{X}^{\perp} \varphi Y-\nabla_{Y}^{\perp} \varphi X .
\end{gathered}
$$

Then we obtain,

$$
[X, Y] \in D^{\perp} \oplus \operatorname{Sp}\left\{\xi_{1}, \ldots, \xi_{s}\right\} \Rightarrow A_{\varphi X} Y=A_{\varphi Y} X .
$$

Conversely

$$
\begin{gathered}
\varphi^{2}([X, Y])=\sum_{i=1}^{s}\left\{2 g(X, \varphi Y) \varphi \xi_{i}+\eta^{i}(Y) \varphi^{2} X-\eta^{i}(X) \varphi^{2} Y\right\} \\
+A_{\varphi X} Y-A_{\varphi Y} X+\varphi\left(\nabla_{X}^{\perp} \varphi Y\right)-\varphi\left(\nabla_{Y}^{\perp} \varphi X\right) \\
{[X, Y]=\sum_{i=1}^{s}\left\{-\eta^{i}(Y) X+\eta^{i}(X) Y\right\}+\sum_{i=1}^{s}\left\{\eta^{k}(X) \xi_{k}-\eta^{k}(X) \xi_{k}\right\}} \\
+\varphi\left(\nabla_{X}^{\perp} \varphi Y\right)-\varphi\left(\nabla_{Y}^{\perp} \varphi X\right)
\end{gathered}
$$

then, we have

$$
[X, Y] \in D^{\perp} \oplus \operatorname{Sp}\left\{\xi_{1}, \ldots, \xi_{s}\right\}
$$

\section{References}

[1] D.E. Blair, Geometry of manifolds with structural group $U(\mathrm{n})$ x $O(\mathrm{~s})$, J. Differ. Geom. 4, 155-167 (1970).

[2] K. Kenmotsu, A class of almost contact Riemannian manifolds, Tohoku Math. J. 24 (1972) 93-103.

[3] M. Kobayashi, Semi-invariant submanifolds of a certain class of almost contact manifolds, Tensor N. S. 43 (1986) 28-36.

[4] S. Goldberg and K. Yano, Globally framed f-Manifolds, Illinois J. Math.15, 456-474 (1971).

[5] S. A. Nirmala and R.C. Mangala, a semi-symmetric non-metric connection on Riemannian manifold, Indiana J. Pure Appl. Math. 23 399-409 (1992).

[6] B. Prasad, Semi-invariant submanifolds of a Lorentzian para-Sasakian manifold, Bull. Malaysian Math. Soc. 21 (1998) 21-26.

[7] B. B. Sinha and A. K. Srivastava, Semi-invariant submanifolds of a Kenmotsu manifold with constant $\varphi$-holomorphic sectional curvature, Indian J. pure appl. Math. 23(11):783-789 (1992).

[8] M. M. Tripathi, A new connection in a Riemannian manifold, Int. Ele.Journal of Geometry Vol.1 No.1 15-24 (2008).

[9] A Turgut Vanli and R. Sari, Generalized Kenmotsu manifolds, Arxiv 1406.1032v1.

[10] J. Vanzura, Almost r-contact structures, Ann. Scuola Norm. Sup. Pisa Sci. Fis. Mat. 26 (1972), 97-115.

[11] K. Yano, On a structure defined by a tensor field f of type $(1,1)$ satisfying $f^{3}+f=0$, Tensor NS., 14, 99-109 (1963) . 